\title{
CONTINUOUS MAPPINGS INTO NONSIMPLE SPACES
}

\author{
BY \\ J. DUGUNDJI
}

1. Introduction. This paper deals with the homotopy classification of the continuous maps of a space $X$ into a nonsimple space $Y$. This problem has been previously discussed by Olum [3], but his approach differs considerably from the one to be presented. Essentially, we first reduce the question to one of classifying certain types of maps into simple spaces, and then use the known apparatus; it is felt that considerable simplification in treatment is achieved.

The procedure used here is the following: Replace $X$ by a suitable covering space $\tilde{X}$ and $Y$ by its universal covering space $\tilde{Y}$. For each homomorphism $h$ of the group $W$ of deck-transformations of $\tilde{X}$ into that of $\tilde{Y}$, take $W$ as operators on $\tilde{X}$ and, through $h$, on $\tilde{Y}$; split the continuous maps of $\tilde{X}$ into $\tilde{Y}$ equivariant with respect to these operators into equivariant homotopy classes. The set of classes so obtained, for all $h$, bear a definite relation to the homotopy classes of the base spaces, so that the problem is reduced to the classification of equivariant maps into a space simple in all dimensions. The classification scheme and theorem of Eilenberg [2] are shown to go over completely for the equivariant maps considered (even with proofs formally unaltered) whenever $X$ is a polytope. It follows from this that Eilenberg's technique for simple $Y$ has a purely mechanical generalization for maps into nonsimple spaces, the only additional requirement being that the set of homomorphisms $\pi_{1}(X) \rightarrow \pi_{1}(Y)$ induced by continuous maps of $X$ into $Y$ be known. In similar fashion, the generalization by Olum [3] of the Eilenberg scheme for maps of arbitrary $X$, when given for simple $Y$ formally generalizes to apply to the nonsimple case.

The paper is divided in three parts. Part I deals with covering spaces, and with the existence, homotopy, and extension properties of equivariant maps; an important role is played by a condition under which a covering space of a subset $A \subset X$ can be embedded into a covering space of $X$ in such a way that the image still covers $A$. In Part II, the relation between the equivariant homotopy classes of maps of suitable covering spaces and the homotopy classes of the base spaces (both the relative and free homotopy cases) are given. Part III shows the Eilenberg procedure and theorem formally valid for the equivariant maps arising in Part II. Two simple applications, for illustrative purposes, are given in this paper.

Received by the editors July 27, 1956. 


\section{Covering SPACES}

2. Notation and preliminaries. All spaces considered here will be Hausdorff, arc-connected, locally 0 -connected, and semi-locally 1 -connected( $\left.{ }^{1}\right)$. Paths are denoted by small Greek letters; $\alpha * \beta$ is the path

$$
\alpha * \beta(t)=\left\{\begin{array}{l}
\alpha(2 t) \\
\beta(2 t-1)
\end{array}\right.
$$

$$
\begin{gathered}
0 \leqq t \leqq 1 / 2 \\
1 / 2 \leqq t \leqq 1
\end{gathered}
$$

and $\alpha^{-1}(t)=\alpha(1-t)$. The element of the fundamental group $\pi_{1}\left(Y, y_{0}\right)$ represented by $\alpha$ is written $\left(^{2}\right)[\alpha]$. If $\gamma$ is a path from $y_{0}$ to $y_{1}, \gamma^{+}: \pi_{1}\left(Y, y_{1}\right) \rightarrow \pi_{1}\left(Y, y_{0}\right)$ is the isomorphism (onto) $\gamma^{+}[\alpha]=\left[\gamma * \alpha * \gamma^{-1}\right]$. The homomorphism $\pi_{1}\left(X, x_{0}\right)$ $\rightarrow \pi_{1}\left(Y, y_{0}\right)$ induced by a (continuous) $\left(^{3}\right)$ map $f:\left(X, x_{0}\right) \rightarrow\left(Y, y_{0}\right)$ is written $f^{+}$.

Let $y_{0} \in Y$ be fixed. $\tilde{Y}(B)$ denotes the covering space of $Y$ corresponding to the subgroup $B \subset \pi_{1}\left(Y, y_{0}\right)$ and $p_{Y}: \tilde{Y}(B) \rightarrow Y$ the projection.

2.1. In all future considerations, base point for the fundamental group of $\tilde{Y}(B)$ will be a point $\tilde{y}_{0} \in p^{-1}\left(y_{0}\right)$ such that $p_{Y}^{+}$maps $\pi_{1}\left(\tilde{Y}(B), \tilde{y}_{0}\right)$ isomorphically onto $B$.

The following implications of the covering homotopy theorem $[6, p .54]$ are frequently used:

2.2. Given $\tilde{y} \in \tilde{Y}(B)$ projecting to a point on $\alpha \subset Y$ there is exactly one path $\tilde{\alpha} \subset \tilde{Y}(B)$ projecting onto $\alpha$ and going through $\tilde{y}$.

2.3. If the paths $\alpha, \beta$ in $Y$ are equivalent (written: $\alpha \sim \beta$ ) any two coinitial covering paths are coterminal and equivalent. For $\alpha$ starting at $y_{0}, \tilde{y}(\alpha)$ denotes the endpoint of that covering path in $\tilde{Y}(B)$ starting at the $\tilde{y}_{0}$ chosen in 2.1.

An $\tilde{f}: \tilde{X}(Q) \rightarrow \tilde{Y}(B)$ is said to cover an $f: X \rightarrow Y$ if $f p_{X}=p_{Y} \tilde{f}$. As a trivial consequence of 2.2 :

2.4. If $\tilde{f}, \tilde{f}^{\prime}: \tilde{X}(Q) \rightarrow \tilde{Y}(ß)$ both cover $f: X \rightarrow Y$, and if $\tilde{f}(\tilde{x})=\tilde{f}^{\prime}(\tilde{x})$ for some $\tilde{x}$, then $\tilde{f}^{\prime}=\tilde{f}$.

$N(B)$ will denote the normalizer $\left(^{4}\right)$ of $B \subset \pi_{1}\left(Y, y_{0}\right)$. The group of decktransformations $\left(^{(}\right)$of $\tilde{Y}(B)$ is isomorphic to $N(B) / B$. The deck-transformation $D_{d}$ corresponding to $d \in N(B) / \otimes$ will always be taken to be the map $D_{d}(\tilde{y})=\tilde{y}\left(\delta * p_{Y} \tilde{\eta}\right)$ where $[\delta]$ is a representative of $d$ and $\tilde{\eta}$ a path from $\tilde{y}_{0}$ to $\tilde{y}$. Note that $D_{d * d^{\prime}}(\tilde{y})=D_{d}\left(D_{d^{\prime}}(\tilde{y})\right)$.

3. Equivariant covering maps. Let $a \subset \pi_{1}\left(X, x_{0}\right), B \subset \pi_{1}\left(Y, y_{0}\right)$ be fixed subgroups, and fix base points $\tilde{x}_{0}, \tilde{y}_{0}$ in $\tilde{X}(Q), \tilde{Y}(B)$ according to 2.1 .

(1) Each point has a nbd $V$ such that any closed path in $V$ is homotopic to a constant in $X$, keeping the endpoints fixed.

(2) The (multiplicative) group operation in $\pi_{1}$ is also denoted by "* ".

(3) All maps are continuous unless explicitly stated otherwise.

$\left({ }^{4}\right) N(B)$ is the largest subgroup containing $B$ in which $B$ is normal.

(†) Also called "covering transformations," "deckbewegungen." 
Let $h: N(Q) / Q \rightarrow N(B) / \otimes$ be a homomorphism. By associating with each $d \in N(Q) / Q$ the deck-transformation $D_{d}$ of $\tilde{X}(Q)$ and $D_{h d}$ of $\tilde{Y}(\mathbb{B}), N(Q) / Q$ will be regarded as a group of operators $\left(^{(}\right)$acting on both $\tilde{X}(Q)$ and $\tilde{Y}(B)$. $N(a) / Q$ operates effectively and fixed-point-free on $\tilde{X}(a)$, but is not necessarily transitive on the fibers.

An $\tilde{f}: \tilde{X}(Q) \rightarrow \tilde{Y}(B)$ equivariant $\left({ }^{7}\right)$ under the action of $N(Q) / Q$ on $\tilde{X}(Q)$ and, through a specified $h: N(Q) / Q \rightarrow N(B) / Q$ on $\tilde{Y}(B)$, is called $h$-equivariant. In at least one case, an $h$-equivariant map is always a covering map:

3.1. If $a$ is normal, any $h$-equivariant $\tilde{f}: \tilde{X}(Q) \rightarrow \tilde{Y}(B)$ covers an $f: X \rightarrow Y$. For in this case, $N(Q)=\pi_{1}\left(X, x_{0}\right)$, so the operators are transitive on each fiber; the formula $f(x)=p_{Y} \tilde{f}(\tilde{x}), \tilde{x} \in p_{X}^{-1}(x)$ therefore defines $f(x)$ uniquely, and its continuity is immediate.

A necessary condition for the existence of $h$-equivariant covering maps is

3.2. THEOREM. Let $h: N(Q) / Q \rightarrow N(B) / @$ be given, and let the h-equivariant $\tilde{f}:\left(\tilde{X}(Q), \tilde{x}_{0}\right) \rightarrow(\tilde{Y}(B), \tilde{y}(\alpha))$ cover an $f:\left(X, x_{0}\right) \rightarrow(Y, y)$. Then $\alpha^{+} f^{+}:(N(Q), a)$ $\rightarrow(N(B), B)$ and $h=h\left(\alpha^{+} f^{+}\right)$, where $h\left(\alpha^{+} f^{+}\right)$is the homomorphism $N(Q) / Q$ $\rightarrow N(B) / B$ induced by $\alpha^{+} f^{+}$.

Proof. Let $[\delta] \in N(Q)$ represent $d \in N(Q) / Q$ and $[\mu] \in N(B)$ represent $h(d)$. Since $D_{d} \tilde{x}_{0}$ can be joined to $\tilde{x}_{0}$ by a path covering $\delta$, it follows from 2.2 that $\tilde{f} D_{d} \tilde{x}_{0}=\tilde{y}(\alpha * f \delta)$. By equivariance, $\tilde{y}(\alpha * f \delta)=D_{h d} \tilde{f}\left(\tilde{x}_{0}\right)=\tilde{y}(\mu * \alpha)$ so $\alpha * f \delta * \alpha^{-1} * \mu^{-1}$ represents an element of $ß$. Both conclusions follow immediately.

The condition given in 3.2 is also sufficient:

3.3. Theorem. Let $f:\left(X, x_{0}\right) \rightarrow(Y, y)$ be given. For each path $\alpha$ from $y_{0}$ to $y$ such that $\alpha^{+} f^{+}:(N(Q), Q) \rightarrow(N(B), \beta)$ there is a unique $h\left(\alpha^{+} f^{+}\right)$-equivariant $\tilde{f}_{\alpha}:\left(\tilde{X}(Q), \tilde{x}_{0}\right) \rightarrow(\tilde{Y}(B), \tilde{y}(\alpha))$ covering $f$. Furthermore, $\tilde{f}_{\alpha}=\tilde{f}_{\beta}$ if and only if $\left[\alpha * \beta^{-1}\right] \in ß$.

Proof. Existence of $\tilde{f}$ is well-known: the covering map is defined by $\tilde{f}(\tilde{x})=\tilde{y}\left(\alpha * f p_{x} \tilde{\xi}\right)$ where $\tilde{\xi}$ is a path in $\tilde{X}(\alpha)$ from $\tilde{x}_{0}$ to $\tilde{x}$, independence of the $\tilde{\xi}$ used coming from $\alpha^{+} f^{+} a \subset \beta$; continuity results from that of $f, p_{X}$ and the local homeomorphism property of $p_{Y}$. To prove equivariance, let $[\delta]$ represent $d \in N(Q) / Q$ and let $\tilde{\xi}$ run from $\tilde{x}_{0}$ to $\bar{x}$; then $\tilde{f} D_{d} \bar{x}=\tilde{y}\left(\alpha * f \delta * f p_{x} \tilde{\xi}\right)$. Since $[\mu]=\left[\alpha * f \delta * \alpha^{-1}\right]$ represents $h\left(\alpha^{+} f^{+}\right)(d)$ and since $\alpha * f \delta * f p_{x} \tilde{\xi} \sim \mu * \alpha * f p_{x} \tilde{\xi}$, 2.3 shows $\bar{f} D_{d} \tilde{x}=\tilde{y}\left(\mu * \alpha * f p_{x} \tilde{\xi}\right)=D_{h d} \tilde{f}(\tilde{x})$ establishing equivariance. The final conclusion results from 2.4 and the observation that $\left[\alpha * \beta^{-1}\right] \in B$ is equivalent to $\tilde{y}(\alpha), \tilde{y}(\beta)$ coterminal.

3.4. REMARK. Notice that $\alpha^{+} f^{+} a \subset B$ alone suffices to prove existence of

(6) A multiplicative group $W$ operates on a space $Z$ if for each $w \in W$ there is given a $w: Z \rightarrow Z$ such that $w_{2} w_{1}(z)=w_{2}\left(w_{1} z\right)$ and $1 z=z$ for every $w_{1}, w_{2} \in W, z \in Z$. If each $w \neq 1$ has no fixed point, $W$ acts fixed-point-free; if $w z=z$ for every $z$ implies $w=1, W$ operates effectively; if for each pair $z_{0}, z_{1} \in Z$ there is a $w$ with $w\left(z_{0}\right)=z_{1}, W$ acts transitively.

(7) If $W$ operates on $X$ and $Y$, an $f: X \rightarrow Y$ is equivariant if $f(w x)=w f(x)$ for all $x \in X$, $w \in W$. 
the covering map. Note further that $\alpha^{+} f^{+}:(N(Q), Q) \rightarrow(N(B), B)$ follows from the condition $\alpha^{+} f^{+} a \subset B$ whenever either (1): $B$ is normal, or (2): $\alpha^{+} f^{+} Q=ß$. This will be used later.

4. Equivariant covering homotopies. Let $Q \subset \pi_{1}\left(X, x_{0}\right)$ be given, and identify $X$ with the subspace $X \times O \subset X \times I$. Since the injection $i^{+}: \pi_{1}(Z \times O$, $\left.z_{0} \times O\right) \rightarrow \pi_{1}\left(Z \times I, z_{0} \times O\right)$ is an isomorphism onto for any arc-connected $Z$, it follows that the covering space of $X \times I$ corresponding to $i^{+} a$ can be taken as $\tilde{X}(a) \times I$, with projection $p_{X \times I}(\tilde{x}, t)=\left(p_{X}(\tilde{x}), t\right)$. The covering space $\tilde{X}(Q)$ can therefore be identified with the subset $\tilde{X}(Q) \times O$. As for base points, note that $p_{X}^{+}\left(\pi_{1}\left(\tilde{X}(a), \tilde{x}_{0}\right)\right)=a$ implies $p_{X \times I}^{+}\left(\pi_{1}\left(\tilde{X}(Q) \times I, \tilde{x}_{0} \times O\right)\right)=i^{+} a$ so the point $\tilde{x}_{0} \times O$ satisfies 2.1 for both $\tilde{X}(a) \times O$ and $\tilde{X}(a) \times I$, whenever $\tilde{x}_{0} \in \tilde{X}(a)$ does; it will be used as base point. Since $i^{+}$induces an isomorphism $N(a) / a$ $\approx N(i+a) / i+a, N(Q) / Q$ can be regarded as the group of deck-transformations of $\tilde{X}(a) \times I$, the transformation corresponding to $d \in N(a) / a$ being $D_{i^{+} d}(\tilde{x}, t)$ $=\left(D_{d}(\tilde{x}), t\right)$.

4.1. Theorem. Let $h: N(Q) / a \rightarrow N(\beta) / \otimes$ be given, and let $f:\left(X, x_{0}\right) \rightarrow(Y, y)$ be covered by an h-equivariant $\tilde{f}:\left(\tilde{X}(Q), \tilde{x}_{0}\right) \rightarrow(\tilde{Y}(B), \tilde{y}(\alpha))$. Then:

4.11. Any homotopy $\phi$ of $f$ can be covered by an h-equivariant homotopy $\tilde{\phi}$ of $\tilde{f}$.

4.12. If $\phi$ is a homotopy $\operatorname{rel} A$, then $\tilde{\phi}$ is $\operatorname{rel} p_{X}^{-1}(A)$.

4.13. If $\mu$ is the path $\mu(t)=(x * t)$ in $X \times I$, then $\tilde{\phi}\left(\tilde{x}_{0} \times 1\right)=\tilde{y}(\alpha * \phi \mu)$.

Proof. Since $\phi^{+} i^{+}=f^{+}$and $N\left(i^{+} Q\right)=i^{+} N(Q)$, it follows that $\alpha^{+} \phi^{+}\left(i^{+} Q\right)$ $=\alpha^{+} f^{+} Q$, and $\alpha^{+} \phi^{+}\left(N\left(i^{+} Q\right)\right)=\alpha^{+} f^{+} N(Q)$. Using the identifications mentioned, and applying $3.2,3.3, \phi$ is covered by an $h\left(\alpha^{+} \phi^{+}\right)$-equivariant $\tilde{\phi}: \tilde{X}(Q) \times I$ $\rightarrow \tilde{Y}(B)$. For $d \in N(Q) / Q, h\left(\alpha^{+} \phi^{+}\right)\left(i^{+} d\right)=h\left(\alpha^{+} f^{+}\right)(d)=h(d)$ according to 3.2 , so the $h\left(\alpha^{+} \phi^{+}\right)$-equivariance of $\widetilde{\phi}$ yields $\widetilde{\phi}\left(D_{d} \tilde{x}, t\right)=\widetilde{\phi} D_{i^{+} d}(\tilde{x}, t)=D_{h d} \tilde{\phi}(\tilde{x}, t)$ showing $\tilde{\phi}$ is $h$-equivariant. Finally, $\tilde{\phi} \mid \tilde{x}(Q) \times O=\tilde{f}$, since $\widetilde{\phi}\left(\tilde{x}_{0}, O\right)=\tilde{f}\left(\tilde{x}_{0}\right)$ by choice of base point and construction, and 2.4 applies. $4.12,4.13$ are immediate.

4.2. REMARK. If $a$ in 4.1 is normal, 4.11 has the converse: any $h$-equivariant homotopy $\operatorname{rel} p_{X}^{-1}(A)$ of $\tilde{f}$ projects to a homotopy rel $A$ in $Y$. This is immediate from 3.1 .

The path traced by the image of $x_{0}$ during the homotopy $\phi$ in 4.1 determines $\widetilde{\phi}\left(\tilde{x}_{0}, 1\right)$. In case $X$ is a polytope $\left({ }^{8}\right)$ it will be shown that the given $f$ can be deformed in such a way that $\tilde{\phi}\left(\tilde{x}_{0}, 1\right)$ is a specified point in $p_{Y}^{-1} \phi\left(x_{0}, 1\right)$. Stated fully,

4.3. Corollary. Let $X$ be a polytope, $h: N(Q) / Q \rightarrow N(B) / @$ a homomorphism, and $f:\left(X, x_{0}\right) \rightarrow(Y, y)$ covered by an h-equivariant $\tilde{f}:\left(\tilde{X}(Q), \tilde{x}_{0}\right) \rightarrow(\tilde{Y}(B)$, $\tilde{y}(\alpha))$. Then there is a $g:\left(X, x_{0}\right) \rightarrow\left(Y, y_{0}\right)$ homotopic to $f$ and an h-equivariant $\tilde{g}:\left(\tilde{X}(Q), \tilde{x}_{0}\right) \rightarrow\left(\tilde{Y}(B), \tilde{y}_{0}\right)$ covering $g$ such that a homotopy of $f$ to $g$ is covered by an h-equivariant homotopy of $\tilde{f}$ to $\tilde{g}$.

(8) By a polytope is meant an arbitrary simplicial $C W$ complex; cf. [7, p. 223]. 
Proof. Define $\phi: X \times O \cup x_{0} \times I \rightarrow Y$ by

$$
\begin{aligned}
& \phi(x, 0)=f(x), \\
& \phi\left(x_{0}, t\right)=\alpha(1-t) .
\end{aligned}
$$

Since $X \times O \cup x_{0} \times I$ is a retract of $X \times I[2$, p. 234], $\phi$ extends over $X \times I$. Set $g(x)=\phi(x, 1)$. By 4.1 , the homotopy $\phi$ is covered by an $h$-equivariant homotopy $\tilde{\phi}$ of $\tilde{f}$. Since $\tilde{\phi}\left(\tilde{x}_{0}, 1\right)=\tilde{y}(\alpha * \phi \mu)=\tilde{y}\left(\alpha * \alpha^{-1}\right)=\tilde{y}_{0}, \tilde{g}(\tilde{x})=\tilde{\phi}(\tilde{x}, 1)$ is the desired map.

5. Embedding of covering spaces and equivariant covering extensions. Throughout this section, $A \subset X$ will denote an arc-connected locally 0 -connected and semi-locally 1 -connected subset of $X$. The question considered is that of embedding a covering space of $A$ into a covering space of $X$ in such a way that the image will project $\left({ }^{9}\right)$ onto $A$. Base point for the fundamental groups of $A$ and $X$ will be at an $a_{0} \in A$.

5.1. Theorem. Let $A \subset X$ and let $i:\left(A, a_{0}\right) \rightarrow\left(X, a_{0}\right)$ be the injection. Let $a \subset \pi_{1}\left(A, a_{0}\right)$ and $B \subset \pi_{1}\left(X, a_{0}\right)$ be subgroups with $a=i^{+^{-1}}(B)$. Then there is a unique homeomorphism $\left({ }^{10}\right) \tilde{\imath}:\left(\tilde{A}(Q), \tilde{a}_{0}\right) \rightarrow\left(\tilde{X}(B), \tilde{a}_{0}^{\prime}\right)$ covering i. If $A \subset X$ is closed, so also is $\tilde{i} \tilde{A}(Q)$ in $\tilde{X}(B)$.

Proof. The existence of $i$ covering $i$ is immediate $\left({ }^{11}\right)$ from 3.4. That $\tilde{i} \tilde{A}(Q)$ is closed in $\tilde{X}(B)$ whenever $A$ is closed in $X$ is a consequence of the existence of $\tilde{i}$ : to show $\tilde{x} \notin \tilde{\imath} \tilde{A}(Q)$ has a nbd not intersecting $\tilde{\imath} \tilde{A}(Q)$ it suffices to consider only the case $p_{X} \tilde{x}=a \in A$ because $A$ is closed. Select a nbd $\tilde{U}(\tilde{x})$ of $\bar{x}$ on which $p_{X}$ is a homeomorphism, and let $U=p_{X} \tilde{U}$. Since $A$ is locally 0 -connected( $\left.{ }^{12}\right)$ there is a nbd $V$ in $X$ with $a \in V \cap A \subset U \cap A$ and any two points in $V \cap A$ can be joined by a path in $V \cap A$. The set $\tilde{U}(\tilde{x}) \cap p p^{-1}(U \cap V)$ is open, contains $\tilde{x}$, and is easily seen to contain no point of $\tilde{\imath} \tilde{A}(a)$. To prove $\tilde{\imath}$ is a homeomorphism, it is sufficient to show $\tilde{\imath}$ is $1-1$; the bicontinuity will follow because $i$ is a local homeomorphism. Let $\tilde{a}_{1} \neq \tilde{a}_{2}$; since $i$ is $1-1$ and $i$ covers $i$, only the case $p_{A} \tilde{a}_{1}=p_{A} \tilde{a}_{2}$ need be considered. Let paths $\tilde{\xi}_{n}$ run from $\tilde{a}_{0}$ to $\tilde{a}_{n}, n=1,2$; the path $p_{A} \tilde{\xi}_{1} * p_{A} \tilde{\xi}_{2}^{-1}$ is closed at $a_{0}$ and since it is not covered by a path closed at $\tilde{a}_{0}$, from $2.1,2.3$ follows that it can not represent an element of $Q$. Because $Q=i^{+^{-1}}(B), i p_{A} \tilde{\xi}_{1} * i p_{A} \tilde{\xi}_{2}$ does not represent an element of $B$, so when lifted to $\tilde{a}_{0}^{\prime}$ will not be closed. Thus $\tilde{\imath}\left(\tilde{a}_{1}\right)=\tilde{y}\left(i p_{A} \tilde{\xi}_{1}\right) \neq \tilde{y}\left(i p_{A} \tilde{\xi}_{2}\right)=\tilde{i}\left(\tilde{a}_{2}\right)$ and the theorem is proved.

$\left(^{9}\right)$ The embedding of $\$ 4$ is a special case of this more general result.

(10) $\tilde{a}_{0}, \tilde{a}_{0}^{\prime}$ are base points in $\tilde{A}(Q), \tilde{X}(B)$ fixed in accordance with 2.1 .

(11) The hypothesis $Q \subset i^{+^{-1}}(B)$ clearly suffices to give existence of a covering $i$. However, because $Q$ may be "too small," $\tilde{i}$ need not be a homeomorphism. Example: $A=S^{1} \subset E^{2}=X$ and $a, B=1$.

(12) Recall that the following two formulations of local 0 -connectedness are equivalent: For each $x$ and nbd $U \supset x$ there is a nbd $V \subset U$ of $x$ such that any two points in $V$ can be joined by a path in (1): $U,(2): V$. 
The case that $B$ is precisely the image of $Q$ is of importance because there is also an isomorphic embedding of the group of deck-transformations of $\tilde{A}(\mathrm{Q})$ into that of $\tilde{X}(B)$. Precisely,

5.2. Theorem. Let $A \subset X$ be closed and $i:\left(A, a_{0}\right) \rightarrow\left(X, a_{0}\right)$ the injection. Assume $a \subset \pi_{1}\left(A, a_{0}\right)$ is such that $i^{+^{-1}}\left(i^{+} Q\right)=a$. Then

5.21. $\tilde{A}(Q)$ can be embedded as a closed subset in $\tilde{X}\left(i^{+} Q\right)$ which projects onto $A$ by $p_{x}$;

5.22. $N(a) / a$ can be embedded as a subgroup in $N\left(i^{+} Q\right) / i^{+} a$ hence can be regarded as a group of operators acting effectively as deck-transformations in $\tilde{X}\left(i^{+} a\right)$;

5.23. $\tilde{A}(a) \subset \tilde{X}\left(i^{+} a\right)$ is stable $\left({ }^{13}\right)$ under the operators $N(a) / a$ in $\tilde{X}\left(i^{+} a\right)$.

\section{Proof. Ad 5.21: See 5.1.}

Ad 5.22: Since $i^{+^{-1}}\left(i^{+} a\right)=a, i^{+}:(N(a), a) \rightarrow\left(N\left(i^{+} a\right), i^{+} a\right)$ follows from 3.4, and that the induced $h\left(i^{+}\right): N(Q) / Q \rightarrow N\left(i^{+} Q\right) / i^{+} Q$ is an isomorphism into from the Noether isomorphism theorem. Identifying $N(a) / Q$ with $h\left(i^{+}\right)[N(a) / a]$, the deck-transformation of $\tilde{X}\left(i^{+} a\right)$ which corresponds to $d \in N(\mathrm{a}) / \mathrm{a}$ is $D_{h\left(i^{+}\right) d}$

Ad 5.23: By 5.22 and 3.2, $\tau$ is $h\left(i^{+}\right)$-equivariant.

The identification of 5.2 leads to an extension theorem:

5.3. Theorem. Let $A \subset X$ be closed, $i:\left(A, a_{0}\right) \rightarrow\left(X, a_{0}\right)$ the injection, and $a \subset \pi_{1}\left(A, a_{0}\right)$ such that $Q=i^{+^{-1}}\left(i^{+} Q\right)$. Let $B \subset \pi_{1}\left(Y, y_{0}\right)$ and $h: N(Q) / Q$ $\rightarrow N(B) / B$ be given. Assume $f:\left(A, a_{0}\right) \rightarrow(Y, y)$ is covered by an h-equivariant $\tilde{f}:\left(\tilde{A}(Q), \tilde{a}_{0}\right) \rightarrow(\tilde{Y}(B), \tilde{y}(\alpha))$ and let $F$ be an extension of $f$ over $X$. Then:

5.31. $\tilde{f}$ has an extension $\tilde{F}$ covering $F$.

5.32. If $i^{+}$is onto, then $h\left(i^{+}\right): N(Q) / Q \approx N\left(i^{+} Q\right) / i^{+} Q$ and $\tilde{F}$ is h-equivariant $\left({ }^{14}\right)$.

5.33. If $B$ is normal, $h$ is extendable to an $\bar{h}: N\left(i^{+} Q\right) / i^{+} Q \rightarrow N(B) / @$ and $\tilde{F}$ is $\bar{h}$-equivariant.

5.34. If $i^{+} Q$ is normal, and $\bar{h}$ fixed, any two $\bar{h}$-equivariant extensions of $\tilde{f}$ coincide on $p_{\mathrm{X}}^{-1}(A)$.

Proof. Ad 5.31: Since $\alpha^{+} f^{+} Q \subset \propto$ by 3.2, and $\alpha^{+} F^{+} i^{+} Q=\alpha^{+} f^{+} Q$, the existence of a covering $\tilde{F}$ results from $3.4 ; 2.4$ applied at $\tilde{x}_{0}$ shows $\tilde{F}$ an extension of $\tilde{f}$.

Ad 5.32: Because $i^{+}$is onto and maps $Q$ onto $i^{+} Q$, it also maps $N(Q)$ onto $N\left(i^{+} a\right)$, hence $h\left(i^{+}\right): N(a) / a \approx N\left(i^{+} a\right) / i^{+} a$. From $\alpha^{+} F^{+} N\left(i^{+} a\right)=\alpha^{+} F^{+} i^{+} N(a)$ $=\alpha^{+} f^{+} N(Q), \tilde{F}$ is $h\left(\alpha^{+} F^{+}\right)$-equivariant. Recalling that $d \in N(Q) / Q$ has been identified with $h\left(i^{+}\right) d$ and observing that $h\left(\alpha^{+} F^{+}\right) h\left(i^{+}\right) d=h\left(\alpha^{+} f^{+}\right) d=h(d)$, the $h\left(\alpha^{+} F^{+}\right)$-equivariance of $\tilde{F}$ shows $\tilde{F} D_{h\left(i^{+}\right) d}=D_{h d} \tilde{F}$, proving $\tilde{F}$ is in fact $h$ equivariant.

(13) If $X$ has operators $W, A \subset X$ is stable if $w A \subset A$ for every $w \in W$.

(14) Note that 4.1 appears as a consequence. Since somewhat less was involved in 4.1 , its proof was given separately. 
Ad 5.33: By normality of $B, N(B)=\pi_{1}\left(Y, y_{0}\right)$ so that the condition $\alpha^{+} F^{+}:\left(N\left(i^{+} Q\right), i^{+} Q\right) \rightarrow(N(B), B)$ is trivially satisfied. Taking $\bar{h}=h\left(\alpha^{+} F^{+}\right)$, 3.3 shows $\tilde{F}$ is $\bar{h}$-equivariant; that $\bar{h}$ is an extension of $h$ follows as in 5.32.

Ad 5.34: The deck-transformations are transitive on each fiber in $\tilde{X}\left(i^{+} Q\right)$, so any point in $p_{X}^{-1}(A)$ is the image of a point in $\tilde{A}(a)$; equivariance gives the desired equality.

5.4. REMARK. It is not difficult to see that 5.31 has the following immediate consequence: If $A$ is a retract of $X$ (recall that $i^{+}$is then an isomorphism into) then for any $a \subset \pi_{1}\left(A, a_{0}\right), \tilde{A}(\alpha)$ is a retract of $\tilde{X}\left(i^{+} Q\right)$, the retraction being equivariant under $N(a) / a$ acting on $\tilde{A}(a)$ and $\tilde{X}\left(i^{+} a\right)$. This slightly generalizes [5].

\section{REDUCTION OF THE ENUMERATION PROBLEM}

Let $A$ be a subset of $X$ and $F: X \rightarrow Y$ a fixed map. Let $Y^{X}(A, F)$ be the set of all (continuous) maps $f: X \rightarrow Y$ satisfying $f|A=F| A$ and ${ }^{15}$ ) divide this set into homotopy classes rel $A$, two maps being in the same class if and only if they are homotopic rel $A$. The enumeration of these classes will be shown reducible to an enumeration of equivariant homotopy classes of maps of a suitable space into one which is simple $[6$, p. 87] in all dimensions.

6. Classification in $Y^{x}\left(x_{0}, y_{0}\right)$ rel $x_{0}$. Let $\tilde{X}, \tilde{Y}$ be the universal covering spaces of $X$ and $Y$, and let $x_{0}, \tilde{x}_{0}, y_{0}, \tilde{y}_{0}$ be fixed base points in these spaces For each homomorphism $h: \pi_{1}\left(X, x_{0}\right) \rightarrow \pi_{1}\left(Y, y_{0}\right)$, denote by $\widetilde{F}(h)$ the set of all $h$-equivariant maps $\tilde{f}:\left(\tilde{X}, \tilde{x}_{0}\right) \rightarrow\left(\tilde{Y}, \tilde{y}_{0}\right)$; it is possible that some of these sets are empty.

6.1. TheOREM. Let $S$ denote the set of all distinct pairs $(h, \tilde{C})$ where $h$ is a homomorphism $\pi_{1}\left(X, x_{0}\right) \rightarrow \pi_{1}\left(Y, y_{0}\right)$ and $\tilde{C}$ an h-equivariant homotopy class $\left.{ }^{16}\right)$ rel $\tilde{x}_{0}$ in $\widetilde{F}(h)$. The homotopy classes rel $x_{0}$ in $Y^{X}\left(x_{0}, y_{0}\right)$ are in 1-1 correspondence $\left({ }^{17}\right)$ with the elements of $S$. For fixed $h$, the subset $(h, \widetilde{C})$ is in 1-1 correspondence with the homotopy classes rel $x_{0}$ of $\left\{f \in Y^{x}\left(x_{0}, y_{0}\right) \mid f^{+}=h\right\}$.

Proof. Observe that any two $f, g \in Y^{X}\left(x_{0}, y_{0}\right)$ with $f^{+} \neq g^{+}$can not be homotopic, since two maps homotopic rel $x_{0}$ induce identical homomorphisms $\pi_{1}\left(X, x_{0}\right) \rightarrow \pi_{1}\left(Y, y_{0}\right)$. The theorem therefore reduces to determining, for each given $h$, the homotopy classes rel $x_{0}$ in $M=\left\{f \in Y^{X}\left(x_{0}, y_{0}\right) \mid f^{+}=h\right\}$. By 3.3, each $f \in M$ is covered by a unique $\tilde{f} \in \tilde{F}(h)$ and conversely, from $3.2,3.1$, each $\tilde{f} \in \tilde{F}(h)$ covers an $f \in M$. According to $4.1,4.2, f \simeq g$ rel $x_{0}$ if and only if $\tilde{f}$ is $h$-equivariantly homotopic to $\tilde{g}$ rel $\tilde{x}_{0}$. The theorem follows at once.

7. Classification in $Y^{x}(A, F)$ rel $A, A \neq \varnothing . A$ is assumed arc-connected,

(15) $A$ may be the null set, $\varnothing . Y^{X}(\varnothing, F)$ is written simply $Y^{x}$; if $A$ consists of a single point $x_{0}$, the symbol $Y^{X}\left(x_{0}, y_{0}\right)$ is used.

(16) And hence rel $p_{x}^{-1}\left(x_{0}\right)$ according to $5.34,4.1$.

(17) A definite correspondence is constructed in the course of the proof. 
locally 0-connected, and semi-locally 1 -connected. Base points for the fundamental groups in $A, X$ and $Y$ are at $a_{0} \in A$ and $y_{0} \in F\left(a_{0}\right) \in Y$.

Let $\mathcal{K}$ be the kernel of $(F \mid A)^{+}: \pi_{1}\left(A, a_{0}\right) \rightarrow \pi_{1}\left(Y, y_{0}\right)$, let $\mathcal{C}$ be the center of $\pi_{1}\left(X, a_{0}\right)$, and let $i^{+}: \pi_{1}\left(A, a_{0}\right) \rightarrow \pi_{1}\left(X, a_{0}\right)$ be the injection. The group

$$
\mathfrak{L}=\Re \cap i^{+^{-1}}(C)
$$

clearly depends only on $X, Y, A$, and $F$.

7.1. LEMMA. $i^{+} \mathfrak{L}$ is a central normal subgroup of $\pi_{1}\left(X, a_{0}\right), i^{+^{-1}}\left(i^{+} \mathcal{L}\right)=\mathfrak{L}$ and $\&$ is a normal subgroup of $\pi_{1}\left(A, a_{0}\right)$.

Proof. The first statement is obvious, and the last an immediate consequence of the second. Since $\mathscr{L} \subset i^{+-1}\left(i^{+} \mathfrak{L}\right)$, only the converse inclusion need be proved. Let $a \in i^{+^{-1}}\left(i^{+} \mathscr{L}\right)$; then $i^{+} a=i^{+} b$ for some $b \in \mathcal{K} \cap i^{+^{-1}}(\mathcal{e})$. Since $b \in \mathcal{K},(F \mid A)^{+} a=F^{+} i^{+} a=F^{+} i^{+} b=(F \mid A)^{+} b=1$ shows $a \in \mathcal{K}$; from $b \in i^{+-1}(\mathbb{C})$ follows $i^{+} a \in \mathcal{C}$, hence $a \in i^{+^{-1}}(\mathcal{C})$ also.

As permitted by $5.2, \tilde{A}(\mathcal{L})$ will be regarded as a closed subset of $\tilde{X}\left(i^{+} \mathcal{L}\right)$ and $\pi_{1}\left(A, a_{0}\right) / \mathfrak{L}$ as a subgroup of $\pi_{1}\left(X, a_{0}\right) / i^{+} \mathfrak{L}$. Base point in $\tilde{A}(\mathfrak{L}), \tilde{X}\left(i^{+} \mathfrak{L}\right)$ is $\tilde{a}_{0} \in p_{\bar{X}}^{-1}\left(a_{0}\right)$.

Let $\tilde{Y}$ be the universal covering space of $Y, \tilde{y}_{0} \in p_{Y}^{-1}\left(y_{0}\right)$ a definite base point, and $h: \pi_{1}\left(A, a_{0}\right) / \mathfrak{L} \rightarrow \pi_{1}\left(Y, y_{0}\right)$ the homomorphism induced by $(F \mid A)^{+}$. The map $f=F \mid A$ is covered by a unique $h$-equivariant $\tilde{f}:\left(\tilde{A}(\mathscr{L}), \tilde{a}_{0}\right) \rightarrow\left(\tilde{Y}, \tilde{y}_{0}\right)$, (3.3).

For each extension $\bar{h}$ of $h$ over $\pi_{1}\left(X, a_{0}\right) / i^{+} \mathcal{L}$, let $\mathcal{F}(\bar{h})$ be the set of all $\bar{h}$-equivariant maps $\tilde{X}\left(i^{+} \mathfrak{L}\right) \rightarrow \tilde{Y}$ coinciding with $\tilde{f}$ on $\tilde{A}(\mathfrak{L})$; some of these sets may be empty.

7.2. THEOREM. Let $\bar{S}$ be the set of all distinct pairs $(\bar{h}, \tilde{C})$ where $\bar{h}$ is an extension of $h$ over $\pi_{1}\left(X, a_{0}\right) / i^{+} \mathfrak{L}$, and $\tilde{C}$ is an $\bar{h}$-equivariant homotopy class rel $\tilde{A}(\mathcal{L})$ in $\tilde{F}(\bar{h})$. The homotopy classes rel $A$ in $Y^{X}(A, F)$ are in $1-1$ correspondence with $\left({ }^{17}\right)$ the elements of $\bar{S}$.

Proof. For each $g \in Y^{x}(A, F)$ there is a corresponding (5.33) couple $\left(h\left(g^{+}\right), \tilde{g}\right)$ where $h\left(g^{+}\right): \pi_{1}\left(X, a_{0}\right) / i^{+} \mathfrak{L} \rightarrow \pi_{1}\left(Y, y_{0}\right)$ is an extension of $h$ and $\tilde{g}$ is an $h\left(g^{+}\right)$-equivariant covering map for $g$. By 3.3, 3.1, this corresondence is $1-1$. Note that if $\tilde{g} \simeq \tilde{g}^{\prime}$ rel $\tilde{A}(\mathscr{L})$ equivariantly in any manner (operators $\left.\pi_{1}\left(X, a_{0}\right) / i^{+} \mathfrak{L}\right)$ then $(5.34) \quad \tilde{g} \simeq \tilde{g}^{\prime}$ equivariantly $\operatorname{rel} p_{X}^{-1}(A)$ so $(4.12) g \simeq g^{\prime}$ rel $A$ and, as in 6.1, $h\left(g^{+}\right)=h\left(g^{+}\right)$; couples with distinct $h\left(g^{+}\right)$therefore cannot be equivariantly homotopic. If $h\left(g^{+}\right)$is fixed, the equivalence of $g \simeq g^{\prime}$ rel $A$ and $\tilde{g} \simeq \tilde{g}^{\prime}$ rel $\widetilde{A}(\mathfrak{L}) h\left(g^{+}\right)$-equivariantly follows from $4.2,4.1$. The theorem is proved.

Observe that if $A=x_{0}$, this reduces to 6.1.

8. Classification in $Y^{P}$, free homotopies. Throughout this section, $P$ is a polytope, $\tilde{P}, \tilde{Y}$ are the universal covering spaces, and $p_{0}, \tilde{p}_{0}, y_{0}, \tilde{y}_{0}$ base points 
in the spaces involved, fixed throughout the discussion. The following lemma plays a central role:

8.1. Lemma. Let $h: \pi_{1}\left(P, p_{0}\right) \rightarrow \pi_{1}\left(Y, y_{0}\right)$ be a given homomorphism, and $\tilde{f}, \tilde{g}:\left(\tilde{P}, \tilde{p}_{0}\right) \rightarrow\left(\tilde{Y}, \tilde{y}_{0}\right)$ h-equivariant maps covering $f, g:\left(P, p_{0}\right) \rightarrow\left(Y, y_{0}\right)$ respectively.

8.11. For any $c$ belonging to the centralizer $\left({ }^{18}\right) Z(h)$ of $h\left(\pi_{1}\left(P, p_{0}\right)\right)$ in $\pi_{1}\left(Y, y_{0}\right), D_{c} \tilde{f}$ is h-equivariant.

8.12. $f \simeq g$ if and only if $\bar{f}$ is h-equivariantly homotopic to $D_{c} \tilde{g}$ for some $c \in Z(h)$.

Proof. Ad 8.11: From $c * h(d)=h(d) * c$ for every $d \in \pi_{1}\left(P, p_{0}\right)$ follows $D_{c} D_{h d}=D_{h d} D_{c}$. Since $\tilde{f} D_{d}=D_{h d} \bar{f}$ one finds $D_{c} \tilde{f} D_{d}=D_{h d} D_{c} \tilde{f}$.

Ad 8.12: An $h$-equivariant homotopy of $\tilde{f}$ to $D_{c} \tilde{g}$ covers (3.1) a homotopy of $f$ to $g$. Conversely, any $\phi: f \simeq g$ is covered (4.1) by an $h$-equivariant homotopy of $\tilde{f}$, and $(4.13) \tilde{\phi}\left(\tilde{p}_{0}, 1\right)=\tilde{y}(\alpha)$ where $\alpha=\phi \mu$. Since $\tilde{\phi} \mid \tilde{P} \times 1$ covers $g$ and sends $\tilde{p}_{0}$ to $D_{[\alpha]} \tilde{y}_{0}$, and since $D_{[\alpha]} \tilde{g}$ does the same, 2.4 shows $\tilde{\phi} \mid \tilde{P} \times 1=D_{[\alpha]} \tilde{g}$. Finally, from $h$-equivariance of $\tilde{g}$ and $D_{[\alpha]} \tilde{g} 3.2$ gives $g^{+}=h$ and $\alpha^{+} g^{+}=h$, showing $[\alpha] \in Z(h)$.

Denote by $\{\theta\}$ the set of classes of conjugate homomorphisms $\pi_{1}\left(P, p_{0}\right)$ $\rightarrow \pi_{1}\left(Y, y_{0}\right)$ and in each conjugacy class $\theta$ select a definite homomorphism $h_{\theta}$. Let $\widetilde{F}\left(h_{\theta}\right)$ be the set of all $h_{\theta}$-equivariant $\tilde{f}: \tilde{P} \rightarrow \tilde{Y}$ (some of these sets may be empty) and let $\left\{\tilde{C}\left(h_{\theta}\right)\right\}$ be the set of $h_{\theta}$-equivariant homotopy classes in $\mathcal{F}\left(h_{\theta}\right)$.

For each fixed $h_{\theta}, Z\left(h_{\theta}\right)$ is taken as a group of operators on $\left\{\tilde{C}\left(h_{\theta}\right)\right\}$ by setting

$$
z(\tilde{C})=\left\{\begin{array}{l}
\text { Element of }\left\{\tilde{C}\left(h_{\theta}\right)\right\} \text { represented by } D_{z} \tilde{f} \\
\text { where } z \in Z\left(h_{\theta}\right) \text { and } \tilde{f} \text { represents } \tilde{C} .
\end{array}\right.
$$

The operation is legitimate because of 8.11 , and because $z(\tilde{C})$ is independent of the representative $\tilde{f}$ selected in $\tilde{C}$. Let $\left\{R\left(h_{\theta}\right)\right\}$ be a system of representatives for this operation: every element of $\left\{\widetilde{C}\left(h_{\theta}\right)\right\}$ is the $Z\left(h_{\theta}\right)$-image of exactly one $R \in\left\{R\left(h_{\theta}\right)\right\}$ under possibly many $z$.

8.2. THEOREM. Let $\tilde{S}$ be the set of all distinct pairs $\left(h_{\theta}, R\right)$ where $R$ is an element of $\left\{R\left(h_{\theta}\right)\right\}$. The free homotopy classes of maps in $Y^{P}$ are in 1-1 correspondence $\left({ }^{17}\right)$ with the elements of $\tilde{S}$.

Proof. For each couple $\left(h_{\theta}, R\right)$ there is, by 4.3 , a representative $h_{\theta}$-equivariant $\tilde{f}_{R h_{\theta}}^{+}:\left(\tilde{P}, \tilde{p}_{0}\right) \rightarrow\left(\tilde{Y}, \tilde{y}_{0}\right)$; let $(3.1) f_{R h_{\theta}}$ be the map it covers. (a): No two distinct $f_{R h_{\theta}}$ are homotopic. This is certainly true if the $h_{\theta}$ are different, since (3.2) $f_{R h \theta}^{+}=h_{\theta}$ and homotopic maps induce conjugate homomorphisms. Assuming the $h_{\theta}$ the same, $f_{R h_{\theta}} \simeq f_{R^{\prime} h_{\theta}}$ gives (8.12) $\tilde{f}_{R h_{\theta}} \simeq D_{c} \tilde{f}_{R^{\prime} h_{\theta}}$ for some $c \in Z\left(h_{\theta}\right)$

(18) The centralizer $Z(Q)$ of the subgroup $Q$ in $\mathcal{Y}$ is the set of all $g \in \mathcal{Y}$ satisfying $a g=g a$ for every $a \in \mathbb{Q}$. $Z(\mathfrak{Q})$ is a normal subgroup of $N(\mathbb{Q})$. 
contradicting that the $\left\{f_{R h_{\theta}}\right\}$ are a system of representatives. (b): Each $f: P \rightarrow Y$ is homotopic to some $f_{R h_{\theta}}$. By 4.3 one can assume $f:\left(P, p_{0}\right) \rightarrow\left(Y, y_{0}\right)$. Let $h_{\theta}$ be the representative chosen in the conjugacy class of $f^{+}$; from 3.2 there is an $h_{\theta}$-equivariant $\tilde{f} \in \tilde{F}\left(h_{\theta}\right)$ covering $f$; the homotopy class of $\tilde{f}$ being represented by $D_{c} \tilde{f}_{R h_{\theta}}$ for some $c, R$, one has $f \simeq f_{R h_{\theta}}$. The theorem is proved.

8.3. REMARK. By using 4.3, observe that instead of operating on the classes $\left\{\tilde{C}\left(h_{\theta}\right)\right\}$ one can operate only on the $h_{\theta}$-equivariant classes rel $\tilde{p}_{0}$ of $h_{\theta}$-equivariant maps $\tilde{f}:\left(\tilde{P}, \tilde{p}_{0}\right) \rightarrow\left(\tilde{Y}, \tilde{y}_{0}\right)$; details are omitted.

\section{Classes of Equivariant maps}

In II, the classification problem for $Y^{X}(A, F)$ was reduced to one of equivariant maps of regular $\left({ }^{19}\right)$ covering spaces. A classification theorem analogous to that of Eilenberg [2, p. 243] will be obtained for such maps. It will be seen that his procedure carries over rather mechanically to the relative homotopy case; knowledge of his paper, or of $[6, \S \S 32-34]$ is assumed.

9. Classification theorems. In this section, $P$ is a polytope, and $Q \subset P$ a fixed subpolytope. $\bar{P}=\widetilde{P}(Q)$ is a regular covering space, $\widetilde{Q}=p^{-1}(Q)$, and $\tilde{Y}$ is the universal covering space of $Y$. Base points in $P, \bar{P}$ are fixed at a definite $q_{0} \in Q$ and $\tilde{q}_{0} \in P^{-1}\left(q_{0}\right)$, and in $Y, \tilde{Y}$ at $y_{0}, \tilde{y}_{0} \in P_{y}^{-1}\left(y_{0}\right)$. The group $W$ $=\pi_{1}\left(P, q_{0}\right) / Q$ is the group of deck-transformations of $\bar{P}$ acting also as decktransformations in $\tilde{Y}$; observe that the operators $W$ in $\bar{P}$ consist only of simplicial maps. By a system of representatives for the $n$-simplexes of $\bar{P}$ is meant a collection $\left\{\tilde{S}^{n}\right\}$ of $n$-simplexes of $\bar{P}$ such that every $n$-simplex of $\bar{P}$ is the $W$-image of exactly one $\widetilde{S}^{n}$ under exactly one $w \in W$; since $\bar{P}$ is regular, distinct $\tilde{S}^{n}$ project to distinct simplexes in $P$ and the union of the projections of the closures is the $n$-skeleton $P^{n}$ of $P$.

9.1. Lemma. Let $\tilde{f}: \breve{Q} \rightarrow \tilde{Y}$ be equivariant $\left.{ }^{20}\right)$. Then $\tilde{f}$ has an equivariant extension over $\breve{Q} \cup \bar{P}^{2}$. Consequently for any two equivariant $\widetilde{F}, \widetilde{G}: \bar{P} \rightarrow \bar{Y}$ satisfying $\tilde{F}|\check{Q}=\widetilde{G}| \check{Q}, \tilde{F} \mid \check{Q} \cup \bar{P}^{1}$ is equivariantly homotopic rel $\bar{Q}$ to $\tilde{G} \mid \check{Q} \cup \bar{P}^{1}$.

Proof. For each $\tilde{p}_{0}$ of a system of representative 0 -simplexes for $\bar{P}$ that is not in $\breve{Q}$ define $\bar{f}\left(\tilde{p}_{0}\right)$ arbitrarily, and extend over $\breve{Q} \cup \bar{P}_{0}$ by equivariance. Since $\tilde{Y}$ is arc-connected, define an extension over each representative 1simplex not in $\overparen{Q}$ and extend by equivariance. Because $\pi_{1}(Y)=0$ the map on the boundary of each of a system of representative 2-simplexes extends over the 2 -simplex, hence there is an equivariant extension $\bar{f}^{\prime}$ over $\breve{Q} \cup \bar{P}^{2}$. The second part is an immediate consequence (see $\$ 4$ ).

Since $\tilde{Y}$ is simple in all dimensions, the operators $W$ also act on the $n$th homotopy group $\pi_{n}(\tilde{Y})$ of $\tilde{Y}\left(=\pi_{n}(Y)\right.$ for $\left.n \geqq 2\right)$ by setting $w[f]=[w f]$ for $[f] \in \pi_{n}(\widetilde{Y})$.

(19) $\tilde{X}(Q)$ is called regular if $Q$ is normal.

(20) Since the operators $W$ are fixed in this discussion, the qualifying " $W$ " in $W$-equivariant is omitted when no confusion arises. 
Now let $\tilde{f}: \breve{Q} \cup \bar{P}^{n} \rightarrow \tilde{Y}$ be equivariant; the obstruction cocycle $c^{n+1}(\tilde{f})$ is defined $[2$, p. 237] and

9.2. $c^{n+1}(\tilde{f})$ is an equivariant $\left.{ }^{21}\right)\left(n+1, \pi_{n}(\tilde{Y})\right)$-cocycle in $\bar{P}-\check{Q}$ (in $\bar{P}$ if $\left.Q=q_{0}\right)$, and $c^{n+1}(\tilde{f})=0$ if and only if $\tilde{f}$ extends equivariantly over $\widehat{Q} \cup \bar{P}^{n+1}$.

The equivariance of $c^{n+1}(\tilde{f})$ follows from

$$
w c^{n+1}(\tilde{f})\left(\tilde{\sigma}^{n+1}\right)=w\left[\tilde{f} \mid \tilde{\sigma}^{\cdot n+1}\right]=\left[w \tilde{f} \mid \tilde{\sigma}^{n+1}\right]=\left[\tilde{f} \mid w \tilde{\sigma}^{\cdot n+1}\right]=c^{n+1}(\bar{f})\left(w \tilde{\sigma}^{n+1}\right) .
$$

The "if" in the second statement is trivial, and "only if" follows by using representative $(n+1)$-simplexes as in 9.1 .

For two equivariant $\tilde{f}, \tilde{g}: \breve{Q} \cup \bar{P}^{n} \rightarrow \tilde{Y}$ satisfying $\tilde{f}\left|\breve{Q} \cup \bar{P}^{n-1}=\tilde{g}\right| \breve{Q} \cup \bar{P}^{n-1}$, the difference cochain $d^{n}(\vec{f}, \tilde{g})[2$, p. 237] is defined and

9.3. $d^{n}(\tilde{f}, \tilde{g})$ is an equivariant $\left(n, \pi_{n}(\tilde{Y})\right)$-cochain in $\bar{P}-\check{Q}$ (in $\bar{P}$ if $Q=q_{0}$ ).

In working with the difference cochain, strong use is made of the following general result [2, p. 234]: If $f, g: Q \cup P^{n} \rightarrow Y$ and $f\left|Q \cup P^{n-1} \simeq g\right| Q \cup P^{n-1}$ rel $Q$, then there is an $f^{\prime}: Q \cup P^{n} \rightarrow Y$ with $f^{\prime} \simeq f$ rel $Q$ and $f^{\prime}\left|Q \cup P^{n-1}=g\right| Q \cup P^{n-1}$. The analog of this for equivariant maps is

9.4. Lemma. Let $n \geqq 2$ and $Q \neq \varnothing$. Let $\bar{f}, \tilde{g}: \breve{Q} \cup \bar{P}^{n} \rightarrow \tilde{Y}$ be equivariant, with $\tilde{f} \mid \breve{Q} \cup \bar{P}^{n-1}$ equivariantly homotopic rel $\bar{Q}$ to $\tilde{g} \mid \breve{Q} \cup \bar{P}^{n-1}$. Then there is an equivariant $\tilde{f}^{\prime}: \bar{Q} \cup \bar{P}^{n} \rightarrow \tilde{Y}$ equivariantly homotopic rel $\bar{Q}$ to $\tilde{f}$ and $\tilde{f}^{\prime} \mid \overleftarrow{Q} \cup \bar{P}^{n-1}$ $=\tilde{g} \mid \breve{Q} \cup \bar{P}^{n-1}$.

Proof. Since $n \geqq 2$, observe that the injection $i^{+}: \pi_{1}\left(Q \cup P^{n}, q_{0}\right) \approx \pi_{1}\left(P, q_{0}\right)$; the covering space of $Q \cup P^{n}$ corresponding to $i^{+^{-1}}(Q)$ can therefore be taken as $\breve{Q} \cup \bar{P}^{n}$, and the operators $W$ are precisely the deck-transformations restricted to $\widetilde{Q} \cup \bar{P}^{n}$. This being a regular covering, $\tilde{f}, \tilde{g}$ cover some $f, g: Q \cup P^{n} \rightarrow Y$ by 3.1 , and $f\left|Q \cup P^{n-1} \cong g\right| Q \cup P^{n-1}$ rel $Q$. By the general result mentioned above, there is a homotopy rel $Q$ of $f$ to an $f^{\prime}: Q \cup P^{n} \rightarrow Y$ with $f^{\prime} \mid Q \cup P^{n-1}$ $=g \mid Q \cup P^{n-1}$, and (4.1) this is covered by an equivariant homotopy rel $\bar{Q}$ of $\tilde{f}$ to some $\tilde{f}^{\prime}: \check{Q} \cup \bar{P}^{n} \rightarrow \tilde{Y}$. Since $Q \neq \varnothing$ and, by $4.13, \tilde{f}^{\prime}\left(\tilde{q}_{0}\right)=\tilde{g}\left(\tilde{q}_{0}\right)$, using the arcconnectedness of $\breve{Q} \cup \bar{P}^{n-1}$ it follows, as in 2.4, that $\tilde{f}^{\prime}\left|\widetilde{Q} \cup \bar{P}^{n-1}=\tilde{g}\right| \widetilde{Q} \cup \bar{P}^{n-1}$.

The procedure in [2] can now be followed, verbatim, leading to the following result:

Let $\tilde{F}: \bar{P} \rightarrow \tilde{Y}$ be a fixed equivariant map, and $\tilde{Y}_{e}^{\bar{P}}(\widetilde{Q}, \tilde{F})$ the set of all equivariant maps of $\bar{P}$ into $\tilde{Y}$ which coincide with $\tilde{F}$ on $\bar{Q}$. For each fixed integer $n \geqq 0$, divide $\tilde{Y}_{e}^{P}(Q, \tilde{F})$ in to equivalence classes, two maps $\tilde{f}, \tilde{g}$ belonging to the same class if and only if $\tilde{f} \mid \widetilde{Q} \cup \bar{P}^{n}$ is equivariantly homotopic rel $\mathscr{Q}$ to $\tilde{g} \mid \breve{Q} \cup \bar{P}^{n}$; each class is called an $n$-homotopy class. The set of $n$-homotopy classes is denoted by $S(n ; \breve{Q})$.

\subsection{Theorem. Let $Q \neq \varnothing$. Then}

9.51. If $n \geqq 2$ and $H_{e}^{i+1}\left(\bar{P}, \check{Q} ; \pi_{i}(\tilde{Y}) ; W\right)=O$ for all $i \geqq n+1$ the elements of

(21) Operators: $W$ on $\bar{P}, \widetilde{Y}$ and $\pi_{n}(\tilde{Y})$. The definitions in $[1$, p. 383] are used. 
$S(n ; \breve{Q})$ are in 1-1 correspondence $\left.{ }^{22}\right)$ with those of the cartesian product

$$
S(n-1, \check{Q}) \times H_{e}^{n}\left(\bar{P}, \check{Q} ; \pi_{n}(\tilde{Y}) ; W\right) .
$$

9.52. If $n \geqq 2$ and $H_{e}^{i}\left(\bar{P}, \breve{Q} ; \pi_{i}(\tilde{Y}) ; W\right)=0$ for all $i \geqq n+1$ the $n$-homotopy classes coincide with the homotopy classes.

9.53. $S(1 ; \check{Q})$ consists of a single element (see 9.1).

If $Q=q_{0}$, a single element, $H_{e}^{i}\left(\bar{P}, \check{Q} ; \pi_{j}(\tilde{Y}) ; W\right)$ is replaced by $H_{l}^{i}\left(\bar{P} ; \pi_{j}(\tilde{Y})\right.$; $W)$ throughout. Observe, also, that if $Y$ is simple in dimension $n$, then $W$ operates simply on $\pi_{n}(\tilde{Y})$ and by $\left[1\right.$, p. 412] $H_{e}^{n}\left(\bar{P} ; \pi_{n}(\tilde{Y}) ; W\right)=H^{n}\left(P, \pi_{n}(Y)\right)$, $n \geqq 2$.

10. Applications. Two simple applications of the results in II and III to the classification of maps into nonsimple spaces are given. The purpose is purely illustrative, and only the relative homotopy cases will be considered here.

The first is to show that the Hurewicz theorem on aspherical spaces is a result of the theory, essentially because the process "kills" the fundamental group.

10.1 (Hurewicz). Let $P$ be a polytope, and let $\pi_{i}(Y)=0$ for all $i \geqq 2$. The homotopy classes rel $p_{0}$ in $Y^{P}\left(p_{0}, y_{0}\right)$ are in 1-1 correspondence with the set of all homomorphisms $h: \pi_{1}\left(P, p_{0}\right) \rightarrow \pi_{1}\left(Y, y_{0}\right)$, two maps being homotopic rel $p_{0}$ if and only if they induce the same homomorphism.

In fact, by $9.5, \widetilde{F}(h)$ (see 6.1 ) consists of a single element for each $h$; it is classical that, in this case, each homomorphism can be induced by a suitable choice of $f$.

The second application gives essentially the same formulation as Olum $[3$, p. 46$]$ in the case of homotopies rel $p_{0}$, but differs somewhat for the homotopies rel $Q$.

10.2. Let $P$ be a polytope, and let $\pi_{i}(Y)=0$ for all $i \neq 1, n$. Denote by $\widetilde{P}, \tilde{Y}$ the universal covering spaces. The homotopy classes rel $p_{0}$ in $Y^{P}\left(p_{0}, y_{0}\right)$ are in 1-1 correspondence with the elements of the direct sum

$$
\sum_{\tilde{h}} H_{e}^{n}\left(\tilde{P}, \pi_{n}(\tilde{Y}) ; h\right)
$$

taken over all homomorphisms $h: \pi_{1}\left(P, p_{0}\right) \rightarrow \pi_{1}\left(Y, y_{0}\right)$ induced by maps of $P$ into $Y$. Two maps $f, g$ are homotopic rel $p_{0}$ if and only if $f^{+}=g^{+}$and $d^{n}(\tilde{f}, \tilde{g}) \sim 0$ $f^{+}$-equivariantly where $\bar{f}, \tilde{g}$ are the $f^{+}$-equivariant covering maps of $f$ and $g$ (see 3.3). Similarly, the homotopy classes in $Y^{P}(Q, F)$ rel $Q$ are in $1-1$ correspondence with the elements of

(22) A correspondence is determined as follows: in each $(n-1)$-homotopy class $s$, select a definite $f_{s}$; then for each $d \in H_{c}^{n}\left(\bar{P}, \breve{Q} ; \pi_{n}(\widetilde{Y}) ; W\right)$ the $n$-homotopy class $(s, d)$ is represented by a map $f$ agreeing with $f_{s}$ on $\breve{Q} \cup \bar{P}^{n-1}$ and with $d^{n}\left(\tilde{f}, \tilde{f}_{s}\right)$ in the cohomology class $d . H_{e}^{n}(\cdot ; G ; W)$ represents the $n$th equivariant cohomology group of - with coefficients $G$ and operators $W$. 


$$
\sum_{\bar{h}} H_{e}^{n}\left(\tilde{P}\left(i^{+} \mathscr{L}\right), p^{-1}(Q) ; \pi_{n}(\tilde{Y}) ; \bar{h}\right)
$$

taken over all extensions $\bar{h}$ of $h\left[(F \mid Q)^{+}\right]: \pi_{1}\left(Q, q_{0}\right) / \mathfrak{L} \rightarrow \pi_{1}\left(Y, y_{0}\right)$ over $\pi_{1}\left(P, p_{0}\right) / i^{+} \mathcal{L}$ induced by continuous maps.

This results from 9.5 with 6.1 and 7.2. The question of which homomorphisms of the fundamental groups are induced by maps of $P$ into $Y$, so well as that of expressing the homotopy condition directly in terms of $f$ and $g$, both involve the Eilenberg-MacLane invariant $k^{n+1}$.

Note, further, that for $n \geqq 2$, the $n$-homotopy classes of equivariant covering maps, and the $n$-homotopy classes of maps of the base spaces are related similarly as in $\$ \$ 6-8$.

\section{BIBLIOGRAPHY}

1. S. Eilenberg, Homology of spaces with operators, Trans. Amer. Math. Soc. vol. 61 (1947) pp. $378-417$. 251.

2. - Cohomology and continuous mappings, Ann. of Math. vol. 41 (1940) pp. 231$1-50$.

3. P. Olum, Obstructions to extensions and homotopies, Ann. of Math. vol. 52 (1950) pp.

4. L. Pontrjagin, Topological groups, Princeton University Press, 1939.

5. C. W. Saalfrank, On the universal covering space and the fundamental group, Proc. Amer. Math. Soc. vol. 4 (1953) pp. 650-653.

6. N. E. Steenrod, The topology of fibre bundles, Princeton University Press, 1951.

7. J. H. C. Whitehead, Combinatorial homotopy I, Bull. Amer. Math. Soc. vol. 55 (1949) pp. 213-245.

University of Southern California,

Los Angeles, Calif. 